\title{
The correlation between systolic and diastolic blood pressure and diastolic parameters in arterial hypertension in the presence of normal systolic function
}

\author{
Snežana Lazić*, Bratislav Lazić \\ Faculty of Medical Science University of Pristina, Kosovska Mitrovica, Kosovo
}

Introduction: Diastolic abnormalities in arterial hypertension are more prominent in the presence of the accompanying left ventricular hypertrophy (LVH). In the hypertrophic left ventricle due to hypertension, the elevated blood pressure exhibits additional unfavorable effect on complex diastolic process in the presence of normal systolic function. $\mathrm{E} / \mathrm{A}$ ratio is considered to be global index of diastolic function.

Objective: To evaluate the relationship between systolic and diastolic blood pressure and E/A ratio in arterial hypertension with left ventricular hypertrophy with normal systolic function.

Method: Standard Doppler echocardiography, blood pressure levels measured by the standard mercury sphygmomanometer 15 minutes before the examination.

Results: This study analyzed 111 subjects with hypertension (65 with concentric LVH and 46 with eccentric LVH).

\section{Received: $10^{\text {th }}$ Apr 2014}

*Address for correspondence: The Faculty of Medical Science University of Pristina, Anri Dinana bb, 38220 Kosovska Mitrovica, Kosovo.

Phone: +381666060569

E-mail: snezana_lazic@yahoo.com
The individuals with ischemic heart disease, valve abnormalities, diabetes mellitus, and heart rhythm and conductance abnormalities were initially excluded from the study. The study inclusion criteria were EF $>50 \%$ and LVH confirmed by echocardiography. The mean EF was $61 \pm 6 \%$; systolic blood pressure $168 \pm 13 \mathrm{mmHg}$; diastolic blood pressure $101 \pm 7$ $\mathrm{mmHg}$; E/A ratio $0.7 \pm 0.13$; IVRT $110 \pm 4.2 \mathrm{~ms}$; and DT $291 \pm 8.9 \mathrm{~ms}$. The study showed negative correlation between SBP and E/A ratio $(r-0.225 ; p<0.05)$ and between $D B P$ and $E / A$ ratio $(r-0.205 ; p<0.05)$. No significant correlation between SBP and DT $(r-0.003 ; p>0.05)$ or between SBP and IVRT $(r+0.42 ; p>0.05)$ was shown. Furthermore, no significant correlation was shown between DBP and DT $(r-0.17 ;>0.05)$ or DBP and IVRT $(r+0.045 ; p>0.05)$.

Conclusion: Arterial hypertension and left ventricular hypertrophy have been associated with $E / A$ ratio $<1$. The observed negative correlation between SBP and DBP and E/A ratio suggests hemodynamic effect of blood pressure on diastolic function. The results of this study alert about possibility of heart failure during the elevated blood pressure attacks with preserved systolic function.

KEYWORDS: arterial hypertension, echocardiography, diastolic function.

CITATION: Cardiol Croat. 2014;9(5-6):166.

\section{Literature}

1. Müller-Brunotte R, Kahan T, Malmqvist K, Edner M; Swedish ibesartan left ventricular hypertrophy investigation vs atenolol (SILVHIA). Blood pressure and left ventricular geometric pattern determine diastolic function in hypertensive myocardial hypertrophy. J Hum Hypertens. 2003;17:841-9. 